\title{
SET OPERATORS AND ASSOCIATED FUNCTIONS
}

\author{
Shyamapada MODAK and Sk SELIM \\ Department of Mathematics, University of Gour Banga \\ P.O. Mokdumpur, Malda 732 103, INDIA
}

\begin{abstract}
The study of two operators local function and the set operator $\psi$ on the ideal topological spaces are likely to be the same as the study of closure and interior operator of the topological spaces. However, they are not exactly equal to the interior and closure operator of the topological spaces. In this context, we introduce two new set operators on the ideal topological spaces. Detailed properties of these two operators are the part of this article. Furthermore, the operators interior (resp. $\psi$ ) and closure (local function) obey the relation $\operatorname{Int}(A)=X \backslash C l(X \backslash A)\left(\right.$ resp. $\left.\psi(A)=X \backslash(X \backslash A)^{*}\right)$. We search the general method of these relations, through this manuscript.
\end{abstract}

\section{Introduction and Preliminaries}

Let $X$ be a set and $\wp(X)$ be the power set of $X$. A sub-collection $\mathcal{I}$ of $\wp(X)$ is said to be an ideal 921 on $X$ if $\mathcal{I}$ has hereditary and finite additivity property. If $\mathcal{I}$ is an ideal on a set $X$ and $\tau$ is the topology on the same set $X$, then the triplicate $(X, \tau, \mathcal{I})$ is said to be an ideal topological space 3 . We, throughout the paper denoted, it by $\mathcal{G}$. Since $\mathcal{G}$ deals with two mathematical structures: ideal $\mathcal{I}$ and topology $\tau$ simultaneously, thus the condition $\tau \cap \mathcal{I}=\{\emptyset\}$ has played important role for the study of the ideal topological space. This condition termed as codense ideal [4] or $\tau$-boundary [5, 17 or Hayashi-Samuel space [3]. Common representation of various types limit points like condensation point, accumulation point, $\omega$-accumulation point of the topological space is the local function $6,7,7,9$ of the ideal topological space $\mathcal{G}$. For a subset $A$ of $X$, the local function is: $A^{*}=\{x \in X \mid U \cap A \notin \mathcal{I}\}$, where $U \in \tau(x)=\{x \in U \mid U \in \tau\}$. For the detail study of the local function, Natkaniec 18 had introduced another set operator which is called $\psi$ operator. This operator is defined as: for an ideal topological space $\mathcal{G}$ and for $A \subseteq X$, $\psi(A)=X \backslash(X \backslash A)^{*}$. This relation is similar to the well know relation of the

2020 Mathematics Subject Classification. Primary 54A05; Secondary 47H04.

Keywords and phrases. Local function, $\psi$ operator, associated function, $\psi_{\mathcal{M}}$ operator.

$\square$ spmodak2000@yahoo.co.in-Corresponding author; skselim2012@gmail.com

(D) 0000-0002-0226-2392; 0000-0002-4226-2004.

C 2021 Ankara University
Communications Faculty of Sciences University of Ankara-Series A1 Mathematics and Statistics 
topological space $(X, \tau): \operatorname{Int}(A)=X \backslash C l(X \backslash A)$, where $A \subseteq X$, and 'Int' and ' $C l$ ' denoted as the interior and closure operator respectively of the topological space. Note that the 'local function' is not a closure operator and the operator $\psi$ is not an interior operator. However, the operator $l(A)=A \cup A^{*}$ makes a closure operator [6, 7, 9] and it induces a topology on $X$. This topology is called *-topology 17,19 on $X$ and it is denoted as $\tau^{*}(\mathcal{I})$, 8, 10, 14, 16, 20] (or simply $\tau^{*}$ ). The closure operator of the $*$-topology is denoted as $C l^{*}$ and the interior operator of the $*$-topology is denoted as $\operatorname{Int}^{*}$. Furthermore, $\operatorname{Int}^{*}(A)=A \cap \psi(A)[2,5,14,16$.

This present paper has divided into two parts: one part is some new type of set operators on the ideal topological spaces and their relations. Characterizations of the Hayashi-Samuel spaces is also included in this part. The another part of this paper is related to the set-theory. Actually through this part we search the generalization of the following relations: $\psi(A)=X \backslash(X \backslash A)^{*}, \operatorname{Int}(A)=X \backslash C l(X \backslash$ $A)$ and $A^{* \mathcal{M}}=X \backslash \psi_{\mathcal{M}}(X \backslash A)[13$ etc.

Before starting main section we need following tools from the literature:

Proposition 1. [8] Let $\mathcal{G}$ be an ideal topological space. The followings are equivalent.

(1) $X^{*}=X$;

(2) $\tau \cap \mathcal{I}=\{\emptyset\}$

(3) If $I \in \mathcal{I}$, then $\operatorname{int}(I)=\emptyset$;

(4) For every $U \in \tau, U \subseteq U^{*}$.

Lemma 2. [14] Let $\mathcal{G}$ be a Hayashi-Samuel space. Then for $A \subseteq X, \psi(A) \subseteq A^{*}$.

Proposition 3. [5, 8] Let $\mathcal{G}$ be an ideal topological space, and $A$ and $B$ be two subsets of $X$. Then the following properties hold:

(1) If $A \subseteq B$, then $A^{*} \subseteq B^{*}$;

(2) $A^{*}=C l\left(A^{*}\right) \subseteq C l(\bar{A})$,

(3) $\left(A^{*}\right)^{*} \subseteq A^{*}$,

(4) $(A \cup B)^{*}=A^{*} \cup B^{*}$,

(5) If $I \in \mathcal{I}$, then $(A \cup I)^{*}=A^{*}=(A \backslash I)^{*}$,

(6) If $U \in \tau^{*}$, then $U \subseteq \psi(U)$.

Definition 4. A set-valued set function $p: \wp(X) \rightarrow \wp(X)$ is said to be grounded (resp. idempotent) if $p(\emptyset)=\emptyset$ (resp. $p(p(A))=p(A))$, where $A \in \wp(X)$.

\section{The operator $\nabla_{1}$}

We define the operator $\nabla_{1}$ on an ideal topological space $(X, \tau, \mathcal{I})$ in the following way: for a subset $A$ of $X, \nabla_{1}(A)=\underline{\vee}(A) \cap \wedge(A)$, where $\underline{\vee}(A)=\psi(A) \backslash A^{*}$ and $\wedge(A)=\psi(A) \backslash A$.

For the ideal topological space $\mathcal{G}$, if $\mathcal{I}=\{\emptyset\}\left(\right.$ resp. $\mathcal{I}=\wp(X)$ ), then $\nabla_{1}(\emptyset)=\emptyset$ (resp. $\psi(A)=X \backslash A$ for any subset $A$ of $X$ ). 
Lemma 5. Let $\mathcal{G}$ be an ideal topological space and $A$ be a subset of $X$. Then $\nabla_{1}(A)=\psi(A) \backslash\left(A^{*} \cup A\right)$.

Proof. $\left.\nabla_{1}(A)=\left(\psi(A) \backslash A^{*}\right) \cap(\psi(A) \backslash A)=\left[\psi(A) \cap\left(X \backslash A^{*}\right)\right] \cap[\psi(A) \cap(X \backslash A))\right]=$ $\psi(A) \cap\left[\left(X \backslash A^{*}\right) \cap(X \backslash A)\right]=\psi(A) \cap\left[X \backslash\left(A^{*} \cup A\right)\right]=\psi(A) \backslash\left(A^{*} \cup A\right)$.

Theorem 6. Let $\mathcal{G}$ be an ideal topological space. Then following statements hold:

(1) for all $A, B \subseteq X, \psi(A \cup B) \supseteq \nabla_{1}(A) \cap \nabla_{1}(B)$.

(2) for all $A \subseteq X, \nabla_{1}(A)=\psi(A) \backslash\left(C l\left(A^{*}\right) \cup A\right)$.

(3) for all $A \subseteq X, \nabla_{1}(A) \supseteq \psi(A) \backslash(C l(A) \cup A)$.

(4) for all $A \subseteq X, \nabla_{1}(A)=\psi(A) \backslash C l^{*}(A)$.

(5) for all $U \in \tau^{*}, \nabla_{1}(U)=\emptyset$.

(6) for all $U \in \tau, \nabla_{1}(U)=\emptyset$.

(7) for all $A \subseteq X, \nabla_{1}(X \backslash A)=\underline{\vee}(A) \cap \bar{\wedge}(A)$, where $\bar{\wedge}(A)=\left(A \backslash A^{*}\right)$.

(8) for all $A \subseteq X, \psi\left(\nabla_{1}(A)\right)=A \cup X^{*}$.

(9) for all $A \subseteq X, \nabla_{1}(A) \in \tau^{*}$.

Proof. 1. We know $\psi(A) \backslash A^{*} \subseteq \psi(A \cup B) \backslash A^{*}$ and $\psi(A) \backslash A \subseteq \psi(A \cup B) \backslash A$ then $\left[\psi(A) \backslash A^{*}\right] \cap[\psi(A) \backslash A] \subseteq\left[\psi(A \cup B) \backslash A^{*}\right] \cap[\psi(A \cup B) \backslash A]$. Therefore $\nabla_{1}(A) \subseteq \psi(A \cup B)$. Similarly $\nabla_{1}(B) \subseteq \psi(A \cup B)$. Hence $\nabla_{1}(A) \cap \nabla_{1}(B) \subseteq \psi(A \cup B)$ i.e., $\psi(A \cup B) \supseteq \nabla_{1}(A) \cap \nabla_{1}(B)$.

2. From Lemma 5. $\nabla_{1}(A)=\psi(A) \backslash\left(A^{*} \cup A\right)$ and from Proposition $3, A^{*}=$ $C l\left(A^{*}\right)$. Therefore $\nabla_{1}(A)=\psi(A) \backslash\left(C l\left(A^{*}\right) \cup A\right)$.

3. From Lemma 5. $\nabla_{1}(A)=\psi(A) \backslash\left(A^{*} \cup A\right)$ and from Proposition $3, C l\left(A^{*}\right) \subseteq$ $C l(A)$. Therefore $\nabla_{1}(A) \supseteq \psi(A) \backslash(C l(A) \cup A)$.

4. From Lemma 2, $\nabla_{1}(A)=\psi(A) \backslash\left(A^{*} \cup A\right)$. This implies that $\nabla_{1}(A)=$ $\psi(A) \backslash C l^{*}(A)$.

5. We have $\nabla_{1}(U)=\left(\psi(U) \backslash U^{*}\right) \cap(\psi(U) \backslash U)$ and $\wedge(U)=\psi(U) \backslash U$. Hence for $U \in \tau^{*}, \nabla_{1}(U) \subseteq \psi(U) \backslash U=\emptyset, U \subseteq \psi(U)[\overline{5}$.

7. $\nabla_{1}(X \backslash A)=\left[\psi(X \backslash A) \backslash(X \backslash A)^{*}\right] \cap[\bar{\psi}(X \backslash A) \backslash(X \backslash A)]=\left[\left(X \backslash A^{*}\right) \backslash(X \backslash\right.$ $\left.A)^{*}\right] \cap\left[\left(X \backslash A^{*}\right) \backslash(X \backslash A)\right]=\left(\psi(A) \backslash A^{*}\right) \cap\left(A \backslash A^{*}\right)=\underline{\vee}(A) \cap \bar{\wedge}(A)$.

8. We have, $\psi\left(\nabla_{1}(A)\right)=X \backslash\left(X \backslash \nabla_{1}(A)\right)^{*}$. Now $X \backslash\left[\psi(A) \backslash C l^{*}(A)\right]=$ $X \backslash\left[X \backslash(X \backslash A)^{*} \backslash C l^{*}(A)\right]=X \backslash\left[X \backslash C l^{*}(A) \cup(X \backslash A)^{*}\right]=C l^{*}(A) \cup(X \backslash A)^{*}=$ $A \cup A^{*} \cup(X \backslash A)^{*}=A \cup[A \cup(X \backslash A)]^{*}=A \cup X^{*}$.

Corollary 7. Let $\mathcal{G}$ be an ideal topological space. Then for any $A \subseteq X, \nabla_{1}(A) \subseteq$ $\psi\left(\nabla_{1}(A)\right)$.

Now we search the answer of the question that any $*$-open set can be expressed as $U=\nabla_{1}(A)$ for some $A \in \wp(X)$. The answer is negative and it is followed by the following example.

Example 8. Let $X=\{a, b\}, \mathcal{I}=\{\emptyset,\{a\}\}$ and $\tau=\{\emptyset, X\}$. Then $\mathcal{G}=(X, \tau, \mathcal{I})$ is an ideal topological space and $\tau^{*}=\{\emptyset, X,\{b\}\}$.

$\nabla_{1}(\emptyset)=\psi(\emptyset) \backslash C l^{*}(\emptyset)=X \backslash X^{*}=\emptyset$. 


$$
\begin{aligned}
& \nabla_{1}(X)=\psi(X) \backslash C l^{*}(X)=X \backslash \emptyset^{*} \backslash X=\emptyset . \\
& \nabla_{1}(\{a\})=\psi(\{a\}) \backslash C l^{*}(\{a\})=X \backslash\{b\}^{*} \backslash\{a\}=X \backslash\{a, b\} \backslash\{a\}=\emptyset . \\
& \nabla_{1}(\{b\})=\psi(\{b\}) \backslash C l^{*}(\{b\})=X \backslash\{a\}^{*} \backslash X=\emptyset . \\
& \text { Therefore, the } * \text {-open set } X \text { can't be expressed as } \nabla_{1}(A) \text { for some } A \in \wp(X) .
\end{aligned}
$$

The following example shows that the operator $\nabla_{1}$ is not grounded:

Example 9. Let $X=\{a, b, c\}, \tau=\{\emptyset, X,\{a, b\},\{c\}\}$ and $\mathcal{I}=\{\emptyset,\{a\},\{c\},\{a, c\}\}$. Then $\psi(\emptyset)=X \backslash X^{*}=X \backslash\{a, b\}=\{c\}, \emptyset^{*}=\emptyset$. Hence, $\nabla_{1}(\emptyset)=\{c\}$. Therefore $\nabla_{1}$ is not grounded.

The following example shows that the operator $\nabla_{1}$ operator is not an idempotent operator.

Example 10. Let $X=\{a, b, c\}, \tau=\{\emptyset, X,\{a, b\},\{c\}\}$ and $\mathcal{I}=\{\emptyset,\{a\},\{c\},\{a, c\}\}$. Let $A=\{b\}$. Then $\psi(A)=X \backslash\{a, c\}^{*}=X \backslash \emptyset=X$, and $A^{*}=\{b\}^{*}=\{a, b\}$. Thus, $\nabla_{1}(A)=\psi(A) \backslash\left(A \cup A^{*}\right)=X \backslash(\{b\} \cup\{a, b\})=X \backslash\{a, b\}=\{c\}$. Now $\psi(\{c\})=X \backslash\{a, b\}^{*}=X \backslash\{a, b\}=\{c\},\{c\}^{*}=\emptyset$. So, $\nabla_{1}\left(\nabla_{1}(A)\right)=$ $\psi(\{c\}) \backslash\left(\{c\}^{*} \cup\{c\}\right)=\{c\} \backslash(\emptyset \cup\{c\})=\{c\} \backslash\{c\}=\emptyset$ and hence $\nabla_{1}\left(\nabla_{1}(A)\right) \neq \nabla_{1}(A)$.

If we consider the collection $\mathcal{I}_{n}=\{A \in \wp(X) \mid \operatorname{Int}(C l(A))=\emptyset\}$ for a topological space $(X, \tau)$, then $\mathcal{I}_{n}$ is an ideal on $X$ and this ideal is called the ideal of nowhere dense sets.

Remark 11. Let $\mathcal{G}$ be an ideal topological space, where $\mathcal{I}=\mathcal{I}_{n}$. Then $\nabla_{1}(A)=\emptyset$.

However, $\nabla_{1}(A)=\emptyset$ is not true always and it is followed by the following example:

Example 12. In Example 10, we consider $A=\{b\}$. Then $\psi(A)=X \backslash\{a, c\}^{*}=$ $X \backslash \emptyset=X$, and $A^{*}=\{b\}^{*}=\{a, b\}$. Thus, $\nabla_{1}(A)=\psi(A) \backslash\left(A \cup A^{*}\right)=X \backslash(\{b\} \cup$ $\{a, b\})=X \backslash\{a, b\}=\{c\}$.

Theorem 13. Let $\mathcal{G}$ be a Hayashi-Samuel space. Then for $A \subseteq X, \nabla_{1}(A)=\emptyset$.

Proof. We have, $\nabla_{1}(A)=\left(\psi(A) \backslash A^{*}\right) \cap(\psi(A) \backslash A)$. Also from Lemma $2, \psi(A) \subseteq A^{*}$. Therefore $\nabla_{1}(A) \subseteq\left(A^{*} \backslash A^{*}\right) \cap\left(A^{*} \backslash A\right) \subseteq \emptyset$. Thus $\nabla_{1}(A)=\emptyset$.

For converse of the theorem we have following:

Theorem 14. Let $\mathcal{G}$ be an ideal topological space. If $\nabla_{1}(A)=\emptyset$, for all $A \in \wp(X)$, then the space $\mathcal{G}$ is Hayashi-Samuel.

Proof. Given that $\nabla_{1}(X)=\emptyset$. Then $\psi(X) \backslash C l^{*}(X)=\emptyset$. So, $\psi(X) \subseteq X \cup X^{*} \subseteq X$. Again, $X \subseteq \psi(X) \subseteq X \cup X^{*} \subseteq X$, since $X$ is open. Thus, $X=X \cup X^{*}$. Therefore, $X=X^{*}$. Thus from Proposition 1, the space $\mathcal{G}$ is Hayashi-Samuel.

Corollary 15. An ideal topological space $\mathcal{G}$ is Hayashi-Samuel if and only if, for each $A \in \wp(X), \nabla_{1}(A)=\emptyset$. 
$\nabla_{1}(A)=\emptyset$, for all $A \in \wp(X)$ is true only when the ideal topological space is Hayashi-Samuel and it is justified by the Example 10.

More simplified Characterization of the Hayashi-Samuel space is:

Theorem 16. An ideal topological space $\mathcal{G}$ is Hayashi-Samuel if and only if $\nabla_{1}(\emptyset)=$ $\emptyset$.

Proof. Let $(X, \tau, \mathcal{I})$ be a Hayashi-Samuel space. Then $\mathcal{I} \cap \tau=\{\emptyset\}$. Now $\nabla_{1}(\emptyset)=$ $\left(\psi(\emptyset) \backslash \emptyset^{*}\right) \cap(\psi(\emptyset) \backslash \emptyset)=\left(X \backslash X^{*}\right) \cap\left(X \backslash X^{*}\right)=X \backslash X^{*}$. Therefore, $\nabla_{1}(\emptyset)=X \backslash X^{*}=\emptyset$ (by Proposition 1).

Again, if $\nabla_{1}(\emptyset)=\emptyset$, then $X \backslash X^{*}=\emptyset$ implies $X \subseteq X^{*}$ and hence $X=X^{*}$. Thus the ideal topological space $\mathcal{G}$ is Hayashi-Samuel.

Theorem 17. Let $\mathcal{G}$ be an ideal topological space. Then for any $A \in \wp(X), X \backslash$ $\nabla_{1}(A)=A \backslash X^{*}$.

Proof. Given that $\nabla_{1}(A)=\left(\psi(A) \backslash A^{*}\right) \cap(\psi(A) \backslash A)$. Therefore, $\nabla_{1}(X \backslash A)=$ $\left(\psi(X \backslash A) \backslash(X \backslash A)^{*}\right) \cap(\psi(X \backslash A) \backslash(X \backslash A))=\left[\left(X \backslash A^{*}\right) \backslash(X \backslash A)^{*}\right] \cap\left[\left(X \backslash A^{*}\right) \backslash(X \backslash A)\right]=$ $\left[X \backslash(A \cup(X \backslash A))^{*}\right] \cap\left(A \backslash A^{*}\right)=\left(X \backslash X^{*}\right) \cap\left(A \backslash A^{*}\right)=\left(A \backslash A^{*}\right) \backslash X^{*}=A \backslash(A \cup X)^{*}=$ $A \backslash X^{*}$.

Remark 18. Given that $\nabla_{1}(A)$ is open for any $A \in \wp(X)$. But the collection $\left\{\nabla_{1}(A) \mid A \in \wp(X)\right\}$ does not form a basis for a topology on $X$. It is justified by the Example 8 .

\section{The OpERATOR $\nabla_{2}$}

We define the operator $\nabla_{2}$ on an ideal topological space $(X, \tau, \mathcal{I})$ in the following way: for a subset $A$ of $X, \nabla_{2}(A)=\underline{\vee}(A) \cap \bar{\wedge}(A)$, where $\underline{\vee}(A)=\psi(A) \backslash A^{*}$ and $\bar{\wedge}(A)=A \backslash A^{*}$.

Lemma 19. Let $\mathcal{G}$ be an ideal topological space. Then $\nabla_{2}(A)=(\psi(A) \cap A) \backslash A^{*}$ for every $A \in \wp(X)$.

Proof. $\nabla_{2}(A)=\left[\psi(A) \backslash A^{*}\right] \cap\left(A \backslash A^{*}\right)=\left(\psi(A) \cap\left(X \backslash A^{*}\right)\right) \cap\left(A \cap\left(X \backslash A^{*}\right)\right)=$ $(\psi(A) \cap A) \cap\left(X \backslash A^{*}\right)=(\psi(A) \cap A) \backslash A^{*}$.

For the ideal topological space $\mathcal{G}$, if $\mathcal{I}=\{\emptyset\}($ resp. $\mathcal{I}=\wp(X))$, then $\nabla_{2}(\emptyset)=\emptyset$ (resp. $\nabla_{2}(A)=A$, for any subset $A$ of $X$ ).

Theorem 20. Let $\mathcal{G}$ be an ideal topological space. Then followings hold:

(1) for any $A \in \wp(X), \nabla_{2}(A)=\operatorname{Int}^{*}(A) \backslash A^{*}$.

(2) for any $A \in \wp(X), \nabla_{2}(A)=\operatorname{Int} t^{*}(A) \backslash A^{*}$ is open in $\tau^{*}$.

(3) $\nabla_{2}(X)=\underline{\vee}(X)$.

(4) if $U \in \tau^{*}$, then $\nabla_{2}(U)=U \backslash U^{*}=\bar{\wedge}(U)$.

(5) if for any $A \subseteq X, \nabla_{2}(A) \subseteq \underline{\vee}(A)$.

(6) for any $A \in \wp(X), \nabla_{2}(X \backslash A)=\nabla_{1}(A)$.

(7) for any $A \in \wp(X), \nabla_{2}(A)=\nabla_{1}(X \backslash A)$. 
(8) $X \backslash \nabla_{2}(X \backslash A)=X \backslash \nabla_{1}(A)$.

(9) $X \backslash \nabla_{2}(X \backslash A)=A \backslash X^{*}$.

Proof. 1. From Lemma $19, \nabla_{2}(A)=(\psi(A) \cap A) \backslash A^{*}$. As $\operatorname{Int}^{*}(A)=\psi(A) \cap A$, $\nabla_{2}(A)=\operatorname{Int}^{*}(A) \backslash A^{*}$.

2. $\nabla_{2}(A)=\operatorname{Int} t^{*}(A) \backslash A^{*}$ is open in $\tau^{*}$, since $\operatorname{Int} t^{*}(A)$ is open in $\tau^{*}$ and $C l^{*}\left(A^{*}\right)=$ $A^{*} \cup A^{* *} \subseteq A^{*} \cup A^{*}=A^{*}$ is closed in $\tau^{*}$.

3. $\nabla_{2}(X)=(\psi(X) \cap X) \backslash X^{*}=\psi(X) \backslash X^{*}=\underline{\vee}(X)$.

4. $\nabla_{2}(U)=(\psi(U) \cap U) \backslash U^{*}=U \backslash U^{*}$, since $U \subseteq \psi(U)$

5. $\underline{\vee}(A)=\psi(A) \backslash A^{*} \supseteq(\psi(A) \cap A) \backslash A^{*}=\nabla_{2}(A)$. Thus $\nabla_{2}(A) \subseteq \underline{\vee}(A)$.

6. $\nabla_{2}(X \backslash A)=\left[\psi(X \backslash A) \backslash(X \backslash A)^{*}\right] \cap\left[(X \backslash A) \backslash(X \backslash A)^{*}\right]=\left[\left(X \backslash A^{*}\right) \backslash(X \backslash\right.$ $\left.A)^{*}\right] \cap(\psi(A) \backslash A)=\left(\psi(A) \backslash A^{*}\right) \cap(\psi(A) \backslash A)=\nabla_{1}(A)$.

Corollary 21. Let $\mathcal{G}$ be an ideal topological space. Then for any $A \subseteq X, \nabla_{2}(A) \subseteq$ $\psi\left(\nabla_{2}(A)\right)$.

We have for any $A \in \wp(X), \nabla_{2}(A)$ is $*$-open, but it is not true that for any *-open set $U$, there is some $A \in \wp(X)$ such that $\nabla_{2}(A)=U$. It is justified by the following example:

Example 22. Consider Example 8.

$\nabla_{2}(\emptyset)=\emptyset, \nabla_{2}(X)=\emptyset, \nabla_{2}(\{a\})=\emptyset$ and $\nabla_{2}(\{b\})=\emptyset$. Thus for $X \in \tau^{*}$, there is no $A \in \wp(X)$ such that $\nabla_{2}(A)=X$.

Theorem 23. Let $\mathcal{G}$ be an ideal topological space. Then for any subset $A$ of $X$, $X \backslash \nabla_{2}(A)=X^{*} \cup\left[X \backslash A \cup A^{*}\right]$.

Proof. We have $\left(\psi(A) \backslash A^{*}\right) \cap\left(A \backslash A^{*}\right)$. Now $X \backslash\left[\left(\psi(A) \backslash A^{*}\right) \cap\left(A \backslash A^{*}\right)\right]=$ $\left[X \backslash\left(\psi(A) \backslash A^{*}\right)\right] \cup\left[X \backslash\left(A \backslash A^{*}\right)\right]=\left[X \backslash\left(X \backslash(X \backslash A)^{*} \backslash A^{*}\right)\right] \cup\left[X \backslash A \cup A^{*}\right]=[X \backslash$ $\left.\left(X \backslash(X \backslash A)^{*} \cup A^{*}\right)\right] \cup\left[X \backslash A \cup A^{*}\right]=[(X \backslash A) \cup A]^{*} \cup\left[X \backslash A \cup A^{*}\right]=X^{*} \cup\left[X \backslash A \cup A^{*}\right]$.

Theorem 24. Let $\mathcal{G}$ be an ideal topological space. Then for any $A \in \wp(X), \nabla_{2}(X \backslash$ $A)=X \backslash A \cup X^{*}$.

Proof. Given that $\nabla_{2}(A)=\left(\psi(A) \backslash A^{*}\right) \cap\left(A \backslash A^{*}\right)$. Now $\nabla_{2}(X \backslash A)=[\psi(X \backslash A) \backslash$ $\left.(X \backslash A)^{*}\right] \cap\left[(X \backslash A) \backslash(X \backslash A)^{*}\right]=\left[\left(X \backslash A^{*}\right) \backslash(X \backslash A)^{*}\right] \cap\left[(X \backslash A) \backslash(X \backslash A)^{*}\right]=$ $\left[X \backslash\left(A^{*} \cup(X \backslash A)^{*}\right)\right] \cap\left[(X \backslash A) \backslash(X \backslash A)^{*}\right]=(X \backslash A) \backslash(X \backslash A)^{*} \backslash\left(A^{*} \cup(X \backslash A)^{*}\right)=$ $\left(X \backslash A \cup(X \backslash A)^{*}\right) \backslash((X \backslash A) \cup A)^{*}=X \backslash\left(A \cup(X \backslash A)^{*}\right) \backslash X^{*}=X \backslash A \cup(X \backslash A)^{*} \cup X^{*}=$ $X \backslash A \cup(X \cup(X \backslash A))^{*}=X \backslash A \cup X^{*}$.

Corollary 25. Let $\mathcal{G}$ be an ideal topological space. Then for any subset $A$ of $X$, $\nabla_{2}(A)=A \backslash X^{*}$.

Proof. From above theorem, $\nabla_{2}(A)=X \backslash(X \backslash A) \cup X^{*}=(X \backslash(X \backslash A)) \cap\left(X \backslash X^{*}\right)=$ $A \cap\left(X \backslash X^{*}\right)=A \backslash X^{*}$.

Corollary 26. Let $\mathcal{G}$ be an ideal topological space. Then for any member $A$ of $\wp(X), \nabla_{2}(A)=X \backslash \nabla_{2}(X \backslash A)$. 
Proof. Obvious from Theorem 20(9) and Corollary 25

Note that the operator $\nabla_{2}$ is grounded as $\nabla_{2}(\emptyset)=\emptyset$. But it is interesting that this is an idempotent operator.

Corollary 27. Let $\mathcal{G}$ be an ideal topological space. Then for any member $A$ of $\wp(X), \nabla_{2}\left(\nabla_{2}(A)\right)=\nabla_{2}(A)$.

Proof. From Corollary 25, $\nabla_{2}(A)=A \backslash X^{*}$. Then $\left(\nabla_{2}\left(\nabla_{2}(A)\right)=\nabla_{2}\left(A \backslash X^{*}\right)=\right.$ $\left(A \backslash X^{*}\right) \backslash X^{*}=A \backslash(X \cup X)^{*}=A \backslash X^{*}=\nabla_{2}(A)$.

If we consider $\mathcal{I}=\mathcal{I}_{n}$ for the ideal topological space $\mathcal{G}$, then $\nabla_{2}(A)=\emptyset$ for every $A \in \wp(X)$. It is not true that $\nabla_{2}(A)=\emptyset$ for any ideal $\mathcal{I}$ on the topological space $(X, \tau)$.

Example 28. Let $X=\{a, b, c\}, \mathcal{I}=\{\emptyset,\{a\}\}$ and $\tau=\{\emptyset, X,\{a\}\}$. Then $\mathcal{G}$ is an ideal topological space. Let $A=\{a\}$. Then $\psi(A)=X \backslash\{b, c\}^{*}=X \backslash\{b, c\}=\{a\}$, and $A^{*}=\{a\}^{*}=\emptyset$. Thus, $\left.\nabla_{2}(A)=(\psi(A) \cap A) \backslash A^{*}\right)=(\{a\} \cap\{a\}) \backslash \emptyset=\{a\} \backslash \emptyset=$ $\{a\}$.

General rule for $\nabla_{2}(A)=\emptyset$ is:

Theorem 29. Let $\mathcal{G}$ be a Hayashi-Samuel space. Then for $A \in \wp(X), \nabla_{2}(A)=\emptyset$.

Proof. We have, $\nabla_{2}(A)=\left(\psi(A) \backslash A^{*}\right) \cap\left(A \backslash A^{*}\right)$. Also from Lemma $2 \psi(A) \subseteq A^{*}$. Therefore, $\nabla_{2}(A) \subseteq\left(A^{*} \backslash A^{*}\right) \cap\left(A \backslash A^{*}\right) \subseteq \emptyset$. Thus $\nabla_{2}(A)=\emptyset$.

For converse of this theorem we have following:

Theorem 30. Let $\mathcal{G}$ be an ideal topological space. If $\nabla_{2}(X)=\emptyset$, for each $A \in$ $\wp(X)$, then the space $\mathcal{G}$ is Hayashi-Samueel.

Proof. Given that $\nabla_{2}(X)=\emptyset$. Then $\psi(X) \cap X \backslash X^{*}=\emptyset$. So, $\psi(X) \backslash X^{*}=\emptyset$ and hence $X \backslash X^{*}=\emptyset$. That is $X=X^{*}$. Thus, from Proposition 1 , the space $\mathcal{G}$ is Hayashi-Samuel.

Corollary 31. An ideal topological space $\mathcal{G}$ is Hayashi-Samuel if and only if $\nabla_{2}(A)=$ $\emptyset$ for all $A \in \wp(X)$.

More specific characterization of the Hayashi-Samuel space is:

Corollary 32. An ideal topological space $\mathcal{G}$ is Hayashi-Samuel if and only if $\nabla_{2}(X)=$ $\emptyset$.

Proof. Suppose that the space $\mathcal{G}$ is Hayashi-Samuel. Then $\nabla_{2}(X)=\psi(X) \cap X \backslash$ $X^{*}=X \backslash X=\emptyset$.

Converse part is obvious from Theorem 30

Remark 33. Given that $\nabla_{2}(A)$ is open in $\tau^{*}$ for each $A \in \wp(X)$. But the collection $\left\{\nabla_{2}(A) \mid A \in \wp(X)\right\}$ does not form a basis for a topology on $X$. It is justified by the Example 22. 


\section{Associated funCtions}

In this section we shall give a general method for the relation $\operatorname{Int}(A)=X \backslash$ $C l(X \backslash A)$.

Definition 34. A set-valued set function $f: \wp(X) \rightarrow \wp(X)$ is said to be associated with a set-valued set function $g: \wp(X) \rightarrow \wp(X)$ if $f(A)=X \backslash g(X \backslash A)$, for each $A \in \wp(X)$.

Suppose a set-valued set function $f: \wp(X) \rightarrow \wp(X)$ is associated with a setvalued set function $g: \wp(X) \rightarrow \wp(X)$, then we called it by $f$ is associated with $g$ on $X$ and it is denoted as $f \sim^{X} g$.

Example 35. (i) For a topological (resp. metrizable) space $(X, \tau)$, the set operator interior is associated with the set operator closure.

(ii) For an ideal topological space $(X, \tau, \mathcal{I})$, the local function ()$^{*}$ is associated with the set operator $\psi$.

(iii) For a $m$-space $(X, \mathcal{M})$, the interior operator mInt is associated with closure operator $\mathrm{mCl}[15]$.

(iv) For a grill $m$-space the operator $\varphi$ is associated with the operator $\psi_{\varphi}[11]$.

(v) Let $\mathcal{F}$ be a filter on $X$. Then $\mathcal{F}-$ Int $(A)$ is associated with $\mathcal{F}-C l$ [12].

(vi) For a grill minimal space $\left(X, \mathcal{M}, \mathcal{G}_{1}\right)$, the operator ()$^{* \mathcal{M}}$ is associated with the operator $\psi_{\mathcal{M}}[13]$.

(vii) Let $\mu$ be a Generalized Topology in $X$ and $\mathcal{H}$ a hereditary class on $X$. Then the operator $\gamma_{\mu}^{\circ}$ is associated with ()$^{\circ}[1]$.

Theorem 36. Let $f \sim^{X} g$ and $f \sim^{X} h$. Then $g \equiv h$.

Proof. Given that for each $A \in \wp(X), f(A)=X \backslash g(X \backslash A)$ and $f(A)=X \backslash h(X \backslash A)$. Thus, $g(X \backslash A)=h(X \backslash A)$, for all $A \in \wp(X)$. Therefore, $g(A)=h(A)$ and hence $g \equiv h$.

Theorem 37. Let $f \sim^{X} g$. Then $g \sim^{X} f$.

Proof. Given that for any $A \in \wp(X), f(A)=X \backslash g(X \backslash A)$. Then $g(A)=X \backslash f(X \backslash$ A). Therefore, $g \sim^{X} f$.

Theorem 38. If $f \sim^{X} g$, then $f \circ g \sim^{X} g \circ f$.

Proof. Given that for any $A \in \wp(A), f(A)=X \backslash g(X \backslash A)$. Then $g \circ f(A)=$ $g(f(A))=g(X \backslash g(X \backslash A))=X \backslash f(X \backslash(X \backslash g(X \backslash A)))=X \backslash f \circ g(X \backslash A)$. Therefore, $g \circ f \sim^{X} f \circ g$ and hence $f \circ g \sim^{X} g \circ f$.

Theorem 39. Suppose a set-valued set function $f: \wp(X) \rightarrow \wp(X)$ is defined as $f(A)=A$, for all $A \in \wp(X)$. Then $f \sim^{X} f$.

Proof. Given that $f(A)=A=X \backslash f(X \backslash A)$. Thus, $f \sim^{X} f$.

Theorem 40. If $f \sim^{X} g$, then $f \circ f \sim^{X} g \circ g$. 
Proof. $f \circ f(A)=f(X \backslash g(X \backslash A))=X \backslash g(g(X \backslash A))=X \backslash g \circ g(X \backslash A)$. So, $f \circ f \sim^{X} g \circ g$.

Theorem 41. If $f \sim^{X} g$ and $f(A)=A$ for all $A \in \wp(X)$. Then $g(A)=A$, for all $A \in \wp(X)$.

Proof. Given that $f(A)=X \backslash g(X \backslash A)$. This implies that $A=X \backslash g(X \backslash A)$. Thus, $g(A)=A$.

Theorem 42. If $f \sim^{X} g$ and $f(A)=X$ for all $A \in \wp(X)$. Then $g(A)=\emptyset$, for all $A \in \wp(X)$.

Proof. Given that $f(A)=X \backslash g(X \backslash A)$. This implies that $X=X \backslash g(X \backslash A)$. Thus, $g(X \backslash A)=\emptyset$. Therefore, for all $A \in \wp(X), g(A)=\emptyset$.

Theorem 43. If $f \sim^{X} g$ and $g \sim^{X} h$, then $f \equiv h$.

Proof. Given that for any $S \in \wp(X), f(S)=X \backslash g(X \backslash S)$ and $g(S)=X \backslash h(X \backslash S)$. Thus $g(X \backslash S)=X \backslash h(S)$ and hence $f(S)=X \backslash(X \backslash h(S))=h(S)$. Therefore, $f \equiv h$.

Theorem 44. Let $f \sim^{X} g$ and $f\left(S_{1} \cup S_{2}\right)=f\left(S_{1}\right) \cup f\left(S_{2}\right)$, for all $S_{1}, S_{2} \in \wp(X)$. Then $g\left(S_{1} \cap S_{2}\right)=g\left(S_{1}\right) \cap g\left(S_{2}\right)$, for all $S_{1}, S_{2} \in \wp(X)$.

Proof. $g\left(S_{1} \cap S_{2}\right)=X \backslash f\left[X \backslash\left(S_{1} \cap S_{2}\right)\right]=X \backslash f\left(\left(X \backslash S_{1}\right) \cup\left(X \backslash S_{2}\right)\right)=X \backslash f(X \backslash$ $\left.S_{1}\right) \cup f\left(X \backslash S_{2}\right)=\left[X \backslash f\left(X \backslash S_{1}\right)\right] \cap\left[X \backslash f\left(X \backslash S_{2}\right)\right]=g\left(S_{1}\right) \cap g\left(S_{2}\right)$.

Corollary 45. Let $f \sim^{X} g$ and $g\left(T_{1} \cup T_{2}\right)=g\left(T_{1}\right) \cup g\left(T_{2}\right)$, for all $T_{1}, T_{2} \in \wp(X)$. Then $f\left(T_{1} \cap T_{2}\right)=f\left(T_{1}\right) \cap f\left(T_{2}\right)$, for all $T_{1}, T_{2} \in \wp(X)$.

Theorem 46. Let $f \sim^{X} g$ and $f(A \cup B)=f(A) \cap f(B)$, for all $A, B \in \wp(X)$. Then $g(A \cap B)=g(A) \cup g(B)$, for all $A, B \in \wp(X)$.

Proof. Given that $g(A \cap B)=X \backslash f(X \backslash A \cap B)=X \backslash f((X \backslash A) \cup(X \backslash B))=$ $X \backslash[f(X \backslash A) \cap f(X \backslash B)]=[X \backslash f(X \backslash A)] \cup[X \backslash f(X \backslash B)]=g(A) \cup g(B)$.

Corollary 47. Let $f \sim^{X} g$. If $f(A \cap B)=f(A) \cup f(B)$, then $g(A \cup B)=g(A) \cap g(B)$.

Corollary 48. Let $f \sim^{X} g$. If $g(A \cap B)=g(A) \cup g(B)$, then $f(A \cup B)=f(A) \cap f(B)$.

Theorem 49. If $f \sim^{X} g$, then $f \circ g \circ f \sim^{X} g \circ f \circ g$.

Proof. Given that $f(A)=X \backslash g(X \backslash A)$ Then $g(f(A))=g(X \backslash g(X \backslash A))=$ $X \backslash f(g(X \backslash A))$. Therefore $f(g(f(A)))=f(X \backslash f(g(X \backslash A)))=X \backslash g(f(g(X \backslash A)))$. Thus, $f \circ g \circ f \sim^{X} g \circ f \circ g$.

Theorem 50. Let $f \sim^{X} g$. Then for $A \in \wp(X), f \circ g(A)=\emptyset$ if and only if $g \circ f(X \backslash A)=X$.

Proof. Given that $\emptyset=f \circ g(A)=X \backslash g \circ f(X \backslash A)$. This implies $g \circ f(X \backslash A)=X$.

Conversely suppose that $g \circ f(X \backslash A)=X$. Then $X \backslash g \circ f(X \backslash A)=\emptyset$. Hence, $f \circ g(A)=\emptyset$. 
Let $f \sim^{X} g$. Then the collection $\{A \subseteq X \mid f \circ g(A)=\emptyset\}$ is denoted as $\mathcal{I}_{f \circ g}$.

Theorem 51. Let $f \sim^{X} g$. If $B \subseteq A \in \mathcal{I}_{f \circ g}$, then $B \in \mathcal{I}_{f \circ g}$.

Proof. Suppose $B \subseteq A \in \mathcal{I}_{f \circ g}$. Then $f \circ g(B) \subseteq f \circ g(A)=X \backslash g \circ f(X \backslash A)=$ $X \backslash X$ (by Theorem $50=\emptyset$. Therefore, $B \in \mathcal{I}_{f \circ g}$.

Theorem 52. Let $f \sim^{X} g$ and $f\left(I_{1} \cap I_{2}\right)=f\left(I_{1}\right) \cup f\left(I_{2}\right)$, for all $I_{1}, I_{2} \in \wp(X)$. Then for $I_{1}, I_{2} \in \mathcal{I}_{f \circ g}, I_{1} \cup I_{2} \in \mathcal{I}_{f \circ g}$.

Proof. Let $I_{1}, I_{2} \in \mathcal{I}_{f \circ g}$. Then $\left.f \circ g\left(I_{1} \cup I_{2}\right)=X \backslash g \circ f\left(X \backslash I_{1} \cup I_{2}\right)\right)=X \backslash g(f(X \backslash$ $\left.\left.I_{1}\right) \cap\left(X \backslash I_{2}\right)\right)=X \backslash g\left(f\left(X \backslash I_{1}\right) \cup f\left(X \backslash I_{2}\right)\right)=X \backslash\left[g \circ f\left(X \backslash I_{1}\right) \cap g \circ f\left(X \backslash I_{2}\right)\right]=$ $\left[X \backslash g \circ f\left(X \backslash I_{1}\right)\right] \cup\left[X \backslash g \circ f\left(X \backslash I_{2}\right)\right]$. Hence by Theorem 50, $I_{1} \cup I_{2} \in \mathcal{I}_{f \circ g}$.

Remark 53. Let $f \sim^{X} g$ and $f\left(I_{1} \cap I_{2}\right)=f\left(I_{1}\right) \cup f\left(I_{2}\right)$, for all $I_{1}, I_{2} \in \wp(X)$. Then $\mathcal{I}_{f \circ g}$ forms an ideal on $X$.

Corollary 54. Let $f \sim^{X} g$ and $g(A \cap B)=g(A) \cup g(B), \forall A, B \in \wp(X)$. Then $\mathcal{I}_{\text {gof }}$ forms an ideal on $X$.

Corollary 55. (i) Let $f \sim^{X} g$ and $f(A \cap B)=f(A) \cup f(B)$, for all $A, B \in \wp(X)$. Then $\left\{A \subseteq X \mid X \backslash A \in \mathcal{I}_{f \circ g}\right\}$ forms a filter on $X$, when $X \notin \mathcal{I}_{f \circ g}$.

(ii) Let $f \sim^{X} g$ and $g(A \cap B)=g(A) \cup g(B)$, for all $A, B \in \wp(X)$. Then $\left\{A \subseteq X \mid X \backslash A \in \mathcal{I}_{g \circ f}\right\}$ forms a filter on $X$, when $X \notin \mathcal{I}_{g \circ f}$.

Remark 56. (i) Above Remark 53 gives a method for obtaining an ideal on a particular set. But this not the only one method for obtaining an ideal on a particular set. As for example: We consider the interior and closure operators of the topological space $(X, \tau)$, then $\{A \subseteq X \mid \operatorname{Int}(C l(A))=\emptyset\}$ forms an ideal. Note that Int $\sim^{X} C l$, but neither $\operatorname{Int}(A \cap B)=\operatorname{Int}(A) \cup \operatorname{Int}(B)$ nor $C l(A \cap B)=C l(A) \cup C l(B)$.

(ii) Further more for an ideal topological space $(X, \tau, \mathcal{I})$, the operator ()$^{*}$ and $\psi$ obey followings:

For $A, B \subseteq X$,

(a) $(A \cup B)^{*}=(A)^{*} \cup(B)^{*}$.

(b) $\psi(A \cap B)=\psi(A) \cap \psi(B)$.

(c) ()$* \sim^{X} \psi$.

But the collection $\left\{A \subseteq X \mid()^{*} \circ \psi(A)=\emptyset\right\}$ (resp. $\left\{A \subseteq X \mid \psi \circ()^{*}(A)=\emptyset\right\}$ ) does not form an ideal on $X$. It is followed by the following examples.

Example 57. Let $X=\{a, b, c, d\}, \tau=\{\emptyset,\{a\}, X\}$ and $\mathcal{I}=\{\emptyset,\{a\}\}$. Then $\psi \circ()^{*}(\emptyset)=\psi\left(\emptyset^{*}\right)=\psi(\emptyset)=X \backslash X^{*}=X \backslash\{b, c, d\}=\{a\} \neq \emptyset$. Thus $\{A \subseteq X \mid$ $\left.\psi \circ()^{*}(A)=\emptyset\right\}$ does not form an ideal on $X$.

Example 58. Let $X=\mathbb{R}$ be the set of reals. Suppose $\mathbb{R}_{u}$ is the usual topology on $X$ and $\mathcal{I}=\{\emptyset\}$ is the ideal on $X$. Consider $A=\mathbb{Q}$ and $B=\mathbb{R} \backslash \mathbb{Q}$. Then $A \cup B=\mathbb{R}$. Now $\psi(A)=\psi(\mathbb{Q})=\mathbb{R} \backslash(\mathbb{R} \backslash \mathbb{Q})^{*}=\mathbb{R} \backslash \mathbb{R}=\emptyset$ and $\psi(B)=\psi(\mathbb{R} \backslash Q)=\mathbb{R} \backslash \mathbb{Q}^{*}=$ $\mathbb{R} \backslash \mathbb{R}=\emptyset$. Thus, $(\psi(A))^{*}=\emptyset=(\psi(B))^{*}$. Again $(\psi(A \cup B))^{*}=(\psi(\mathbb{R}))^{*}=\mathbb{R}^{*}=\mathbb{R}$. Thus $\left\{A \subseteq X \mid()^{*} \circ \psi(A)=\emptyset\right\}$ does not form an ideal on $X$. 
Authors Contribution Statement Shyamapada Modak: Supervision, Writing, Reviewing and Editing, Conceptualization, Methodology. Sk Selim: Data Creation, Writing, Draft preparation, Investigation.

Declaration of Competing Interest The authors declare that there is no competing financial interests or personal relationships that influence the work in this paper.

Acknowledgment The authors are thankful to the referee and the editors for their valuable comments and suggestions.

\section{REFERENCES}

[1] Al-Omari, A., Modak, S. and Noiri T., On $\theta$-modifications of generalized topologies via hereditary classes, Commun. Korean Math. Soc. 31(4) (2016), 857-868. https://doi.org/ 10.4134/CKMS.c160002

[2] Bandhopadhya, C. and Modak, S., A new topology via $\psi$-operator, Proc. Nat. Acad. Sci. India, Sect. A Phys. Sci. 76(A)(IV) (2006), 317-320.

[3] Dontchev, J., Idealization of Ganster-Reilly decomposition theorems, arXIV:math. Gn/9901017v1 [math.GN], 5 Jan 1999.

[4] Dontchev, J., Ganster, M. and Rose, D., Ideal resolvability, Topology Appl. 93 (1999), 1-16. https://doi.org/10.1016/S0166-8641(97)00257-5

[5] Hamlett, T. R. and Janković, D., Ideals in topological spaces and the set operator $\Psi$, Boll. Un. Mat.Ital. 4-B(7) (1990), 863-874.

[6] Hashimoto, H., On the *-topology and its applications, Fund. Math. 91 (1976), 5-10.

[7] Hayashi, E., Topologies defined by local properties, Math. Ann. 156 (1964), 205-215.

[8] Janković, D. and Hamlett, T. R., New topologies from old via ideals, Amer. Math. Monthly 97 (1990), 295-310, https://doi.org/10.1080/00029890.1990.11995593

[9] Kuratowski, K., Topology, Vol. I, New York, Academic Press, 1966.

[10] Modak, S., Some new topologies on ideal topological spaces, Proc. Natl. Acad. Sci., India, Sect. A Phys. Sci. 82(3) (2012), 233-243 https://doi.org/10.1007/s40010-0120039-3

[11] Modak, S., Operators on grill $M$-space, Bol. Soc. Paran. Mat. 31(2) (2013), 101-107.https: //doi.org/10.5269/bspm.v31i2.15547

[12] Modak, S., Grill-filter spacs, J.Indian Math. Soc. 80(3-4) (2013), 313-320.

[13] Modak, S., Minimal spaces with a mathematical structure, J. Assoc. Arab Univ. Basic Appl. Sci. 22 (2017), 98-101, https://doi.org/10.1016/j.jaubas.2016.05.005

[14] Modak, S. and Bandyopadhyay, C., A note on $\psi$-operator, Bull. Malays. Math. Sci. Soc. 30(1) (2007), 43-48.

[15] Modak, S., Garai, B. Mistry, S., Remarks on ideal $M$-space, Ann. Univ. Oradea Fasc. Mat. 19(1) (2012), 207-215.

[16] Modak, S. and Noiri, T., Connectedness of ideal topological spaces, Filomat 29(4) (2015), 661-665 https://doi.org/10.2298/FIL1504661M

[17] Newcomb, R. L., Topologies which are compact modulo an ideal, Ph. D. Dissertation, Univ. of Cal. at Santa Barbara (1967).

[18] Natkaniec, T., On I-continuity and I-semicontinuity points, Math. Slovaca 36(3) (1986), 297312 .

[19] Samuel, P., A topology formed from a given topology and ideal, J. London Math. Soc. 10 (1975), 409-416. 
[20] Selim, Sk., Islam., Md. M. and Modak, S., Characterizations of Hayashi-Samuel Spaces via Boundary Points, Commun. Adv. Math. Sci. 2(3) (2019), 219-226 https://doi.org/10. $33434 /$ cams.546925

[21] Vaidyanathswamy, R., The localization theory in set-topology, Proc. Indian Acad. Sci. 20 (1945), 51-61 https://doi.org/10.1007/BF03048958 